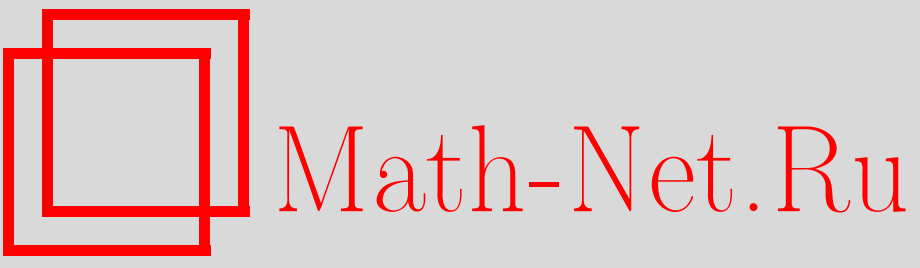

О. В. Шварцман, О рефлективности групп Бъянки, УМН, 1998, том 53, выпуск 6, 271-272

DOI: https://doi.org/10.4213/rm107

Использование Общероссийского математического портала Math-Net.Ru подразумевает, что вы прочитали и согласны с пользовательским соглашением

http://www . mathnet.ru/rus/agreement

Параметры загрузки:

IP : 3.82 .47 .9

26 апреля 2023 г., 15:09:11 


\section{О РЕФЛЕКТИВНОСТИ ГРУПП БЪЯНКИ}

\section{О. В. ШВАРцМАН}

Пусть $O m$ - кольцо целшхх чисел мнимого квадратичного поля $K_{m}=\mathbb{Q}(\sqrt{-m}), m$ - свободное от квадратов натуральное число, $C l_{m}$ - группа классов дивизоров поля $K_{m}$, и $h_{m}$ - число классов. Через $\tau$ обозначим инволюцию комплексного сопряжения на группе $P G L_{2}\left(O_{m}\right)$. Полупрямое произведение $B_{m}=P G L_{2}\left(O_{m}\right) \rtimes\langle\tau\rangle$ назовем группой Ббянки. Группа $B_{m}$ реализуется как дискретная группа движений гиперболического пространства $H^{3}$, и вопрос о ее рефлективности (см. ниже) исследовался в работах $[1]-[3]$. В [1] доказано, что если группа $B_{m}$ реффлективна, то $C l_{m}$ - элементарная 2-группа. Из одного этого необходимого условия уже следует нерефлективность групп $B_{m}$ для всех достаточно больших $m$. Наша цель - получить реальную оценку $m \leqslant 1555$, доказав следующую теорему.

Теорема. Если группа $B_{m}$ рефлективна, mо $h_{m} \leqslant 4$.

Оценка теоремы неулучшаема, как показывает пример рефлективной группы $B_{21}$ [2] В [3] доказано, что если $m \equiv 1,2(\bmod 4)$, то группа $B_{m}$ рефлективна только при $m=1,2,5,6,10,13,21$. Поэтому теорема будет доказана при условии, что $m \equiv 3(\bmod 4)$. После этого вопрос о рефолективности останется открытым для 43 значений $m$, максимальное из которых равно 1555 [4].

1. Пусть $M$ - решетка над $\mathbb{Z}$ с заданным на ней скалярным произведением. Примитивный вектор $x$ из $M$ назьвается $k$-корнем решетки, если $x^{2}=k>0$, и решетка $M$ переходит в себя при отражении $r_{x}$ относительно гиперплоскости, ортогональной вектору $x$ в пространстве $V=M \otimes \mathbb{R}$. Отражение $r_{x}$ назовем $k$-отражением решетки. Если $x$ - корень решетки, то через $M_{x}$ обозначим подрешетку $M \cap(\mathbb{R} x)^{\perp}$. Условимся через $[A]$ обозначать решетку $\mathbb{Z}^{n}$ вместе со скалярньм произведением, заданным на ней с помощью матрицы $A$. Рассмотрим четную гиперболическую решетку ранга 4:

$$
L=\left[\begin{array}{ll}
0 & 1 \\
1 & 0
\end{array}\right] \perp\left[\begin{array}{cc}
2 & 1 \\
1 & \frac{m+1}{2}
\end{array}\right]
$$

(ортогональная сумма), и пусть $K$ - конус всех векторов с отрицательным квадратом в пространстве $V=L \otimes \mathbb{R}$. Выберем одну из его связных компонент $K_{+}$и обозначим через $G$ группу всех ортогональных преобразований пространства $V$, сохраняющих решетку $L$ и конус $K_{+}$. В линейной модели гиперболического пространства группа $G$ является дискретной группой движений $H^{3}$ c некомпактной фундаменталшной областью конечного объема. Группа Бъянки $B_{m}$ вкладываестя в $G$ в виде нормальной подгруппы конечного индекса $\Gamma$ [1]. Пусть $\Gamma_{r}-$ (нормальная) подгруппа в $\Gamma$, порожденная всеми отражениями из $\Gamma$. Реффлективность группы Бъянки означает, что индекс $\left[\Gamma: \Gamma_{r}\right]$ конечен.

2. Здесь собраны необходимые технические леммы.

Лемма 1. Группа $\Gamma_{r}$ содержит только 2- и 2 m-отражения, и все такие отражения из группь $G$ принадлежат группе $\Gamma_{r}$.

Лемма 2. Пусть $x-2$ - или $2 m$-корень решетки L. Тогда а) группа автоморфизмов решетки $L_{x}$ не содержит әлементов порядка 4; б) любой автоморфизм решетки $L_{x}$ порядка 3 есть произведение двух ее 2-отражений.

Лемма 3. Решетка L не содержит трех попарно ортогональных корней одинаковой длиньь.

3. Через $B(C)$ обозначим множество бесконечно удаленных вершин фундаментального многогранника Кокстера $C$ группы $\Gamma r$.

Лемма 4. Группа $G / \Gamma_{r}$ действует транзитивно на множестве $B(C)$. 
ДокАЗАТЕЛЬСтво. Пусть $e-$ примитивный изотропньй вектор в $L$, соответствующий бесконечно удаленной вершине. Включим $е$ в унимодулярную гиперболическую подрешетку $\langle e, f\rangle=$ $\left[\begin{array}{ll}0 & 1 \\ 1 & 0\end{array}\right]$. Тогда

$$
L=\left[\begin{array}{ll}
0 & 1 \\
1 & 0
\end{array}\right] \perp M
$$

где $M$ - четная бинарная евклидова подрешетка с определителем $m$. Так как стабилизатор бесконечно удаленной вершины в группе $\Gamma_{r}$ порожден отражениями, то решетка $M$ содержит 2- или $2 m$-корни. Но тогда $M=\left[\begin{array}{cc}2 & 1 \\ 1 & \frac{m+1}{2}\end{array}\right]$, и наше утверждение следует из теоремы Витта.

Лемма 5. Пусть $x \in B(C)$. Тогда а) при $m>3$ фундаментальная область стабилизатора $\left(\Gamma_{r}\right)_{x}$ на орисфере с чентром в $x$ является прямоугольником, одна пара параллельных сторон которого высекается 2-, а другая - 2m-отражениями; б) стабилизатор $G_{x}$ содержит осевую симметрию второго порядка $\varphi_{x}$, не лежащую в $\left(\Gamma_{r}\right)_{x}$.

ДокАЗАТЕЛЬСтво пункта б). В базисе, где

$$
L=\left[\begin{array}{ll}
0 & 1 \\
1 & 0
\end{array}\right] \perp\left[\begin{array}{cc}
2 & 1 \\
1 & \frac{m+1}{2}
\end{array}\right],
$$

можно выбрать

$$
\varphi_{x}=\left(\begin{array}{cccc}
1 & \frac{m+1}{4} & 1 & \frac{m+1}{2} \\
0 & 1 & 0 & 0 \\
0 & 0 & -1 & 0 \\
0 & -1 & 0 & -1
\end{array}\right)
$$

4. Доказательство теоремы. Группа $G$ есть полупрямое произведение $G=\Gamma_{r} \rtimes T$ группы $\Gamma_{r}$ и конечной группы $T$ симметрий многогранника $C$. Пусть $T^{+}{ }_{-}$подгруппа $T$, состоящая из собственных движений. Если $t \in T^{+}$- элемент порядка 3 , то его неподвижная ось не может проходить ни через ребро, ни через вершину (последнее запрещено леммами 3 и 5 ). Но тогда она пересекает внутренность некоторой грани, и элемент $t$ переводит эту грань в себя. Но это противоречит утверждению леммы 2 , пункт б). Аналогично исключаем присутствие в $T^{+}$элементов порядка 4. Следовательно, $T^{+}{ }_{-}$- абелева 2 -группа, и ее порядок $\left|T^{+}\right| \leqslant 4$, а порядок $|T| \leqslant 8$. Группа $T$ транзитивна на множестве $B(C)$, и порядок стабилизатора любой вершины $\geqslant 2$ по лемме 5. Поэтому $|B(C)| \leqslant 4$. Но тогда и число классов Г-эквивалентных бесконечно удаленных вершин также не превышает четырех. С другой стороны, их число равно $h_{m}[1]$.

Работа выполнена при финансовой поддержке Российского фонда фундаментальных исследований (грант № 97-010-00783) и SFB-343 (Университет г. Билефельд).

\section{СПИСОК ЛИТЕРАТУРЫ}

[1] Винберг Э.Б. // Вопросы теории групп и гомологической алгебры. Ярославль, 1987. C. 121-127. [2] Шайхеев М. К. // Вопросы теории групп и гомологической алгебры. Ярославль, 1987. С. 127-133. [3] Шварцман О. В. // Вопросы теории групп и гомологической алгебры. Ярославль, 1987. С. 134-139. [4] Cohen H. A Course in Computational Algebraic Number Theory. Berlin: Springer-Verlag, 1993. 